\title{
Amalgam alternatives: replacing the irreplaceable?
}

\section{No more amalgams: Use of amalgam and amalgam alternative materials in primary dental care} Br Dent J 2018; 225: 171-176; http://dx.doi.org/10.1038/sj.bdj.2018.538

Change is the only constant. This ancient expression still rings true in our dynamic and modern age. Our daily lives are constantly subject to transformation and the field of dentistry is certainly no exception. The Minamata Treaty and plans for the discontinuation of amalgam marks a significant change taking place in the dental world.

Amalgam is considered one of the hallmark materials of restorative dentistry and it is clear to see why; its longevity, affordability, and ease of manipulation have made it universally popular amongst practitioners. This study aims to highlight what changes must be made to training and research for practitioners to transition seamlessly into amalgam-free practices by answering two key questions: What are the current applications of amalgam and its alternatives in primary dental care and what are the attitudes of dentists in relation to using resin composites as an alternative?

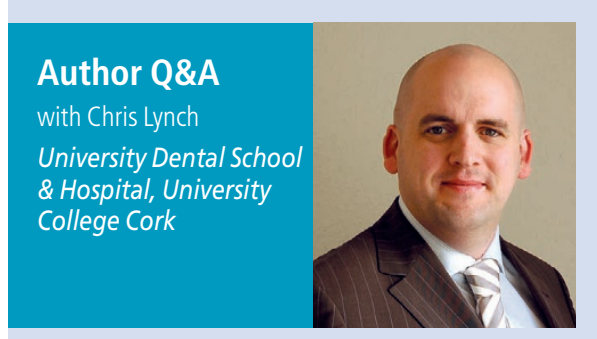

What was the key learning from your study? Our study, of mainly UK-trained, primary care practitioners working in Wales with NHS contracts, shows that amalgam is routinely placed for adult patients. We are very grateful to the 270 dentists who participated in this study, which highlighted that barriers moving from amalgam to resin composite for the restoration of posterior teeth were mainly centred on the financing of NHS care and time implications.

What are the implications for practice? Against a backdrop of the Minamata Treaty, there will be increased pressure on dentists to reduce their routine use of amalgam - as well as an emphasis on practising safe amalgam
The responses highlighted unfavourable financial implications for amalgam alternatives, with participants predicting a $57.5 \%$ increase in NHS fees to accommodate for the posterior placement of resin composite. Amalgam alternatives were also perceived to be very costly with respect to time. Participants predicted the placement of a resin composite in a moderately deep two-surface proximal-occlusal cavity would take them 1.61 times longer on average compared to amalgam. With time being one of the most valuable resources in NHS dentistry, composite restorations may be considered a financially unfeasible option.

Additionally, favourable clinical outcomes were noted with amalgam restorations; $42.6 \%$ agreed or strongly agreed that there was a lower incidence of post-operative sensitivity in patients with amalgam restorations compared to posterior resin composites. Researchers found that $73.3 \%$ of respondents reported that they often or always

disposal. It is estimated that by 2030 , dental amalgam will be the greatest source of environmental mercury - remember this is not just linked to use in a dental surgery, but to other sources, such as crematoria emissions. It is all too easy to conceive that the use of dental amalgam may no longer be tolerated by an environmentally conscious public. We also have to remember that despite the sustained use of amalgam in the $\mathrm{UK}$, there continues to be reduction in the use of amalgam in the rest of the world, and the availability of amalgam may become a challenge over the next 10 years. The risk is that we would be required to eliminate amalgam use over a short period of time, which could cause a crisis amongst the profession.

What challenges do you expect to see with the phasing out of amalgam?

The main challenges, based on the findings of this study, are time and money. Respondents highlighted that resin composites take longer to place in posterior teeth and are more expensive. Another colleague who has encouraged me in this area over the years, Professor Trevor Burke, has argued that the chief dental officers should use amalgam when two or more posterior restorations are required for an NHS-funded adult. However, interestingly, in scenarios involving children and privately funded adults, only $27 \%$ reported amalgam as the material of choice. This may be evidence that NHS funded care may be restricting increased resin use in primary care settings.

This research has highlighted that the material of choice in posterior restorations is influenced by many factors namely funding, time expenditure, experience, clinical outcomes, and pre-existing attitudes. The authors emphasise the need for further development of research into alternative materials and optimising current techniques if practices are to be successfully independent of amalgam. Most importantly perhaps, a shift in attitude is required and the first step may be to remember that composite resins are alternatives, not substitutes for amalgam.

By Arefeh Ahmadifard

look to fund the routine placement of bulk-fill resin composites for NHS patients. This is quite a sensible approach, particularly in light of the increasing evidence base for bulk-fill resin composites.

There is also a need for sign-up to a phasedown, and ultimately phase-out, of amalgam, by stakeholders such as practitioners, funders of NHS dentistry, chief dental officers and postgraduate dental deans. The need for training in this area is important: while our recent surveys of teaching programmes highlight that dental students place twice as many posterior composites than amalgam, it has not always been in the case. Surveys of training programmes from the time when I graduated from dental school (1999) show that as few as 1-in-10 students gained clinical experience in placing posterior resin composites at dental school. The implication of this (assuming no further training or CPD) is that there are cohorts of dentists in their mid-40s and older who do not have routine experience in placing posterior resin composites - efforts will be needed to address their training needs. 